\title{
Efeito de aderências dirigidas em anastomoses cólicas isquêmicas em ratos ${ }^{1}$
}

\author{
Effect of conducted adhesions on ischemic colic anastomoses in rats
}

\author{
Miki Mochizuki², Feng Chui Wu ${ }^{3}$, Cláudio Saddy Rodrigues Coy ${ }^{4}$, Maria de Lourdes Setsuko Ayrizono ${ }^{5}$, Juvenal Ricardo \\ Navarro Góes ${ }^{6}$, João José Fagundes ${ }^{7}$
}

1. Trabalho realizado no Laboratório de Técnica Cirúrgica do Núcleo de Medicina e Cirurgia Experimental da Universidade Estadual de Campinas - UNICAMP.

2. Aluno de Mestrado em Cirurgia - Serviço de Coloproctologia da Faculdade de Ciências Médicas da UNICAMP.

3. Pesquisador e aluno de pós-doutorado - Departamento de Cirurgia - Serviço de Coloproctologia da Faculdade de Ciências Médicas da UNICAMP e Coordenador da área médica do LABI-UNIOESTE / Foz do Iguaçu.

4. Professor Doutor da Disciplina de Moléstias do Aparelho Digestivo do Departamento de Cirurgia - Serviço de Coloproctologia da Faculdade de Ciências Médicas da UNICAMP.

5. Professora da Disciplina de Moléstias do Aparelho Digestivo do Departamento de Cirurgia - Aluna de Doutorado em Cirurgia - Serviço de Coloproctologia da Faculdade de Ciências Médicas da UNICAMP.

6. Professor Associado e Chefe do Departamento de Cirurgia da Faculdade de Ciências Médicas da UNICAMP.

7. Professor Doutor da Disciplina de Moléstias do Aparelho Digestivo do Departamento de Cirurgia - Serviço de Coloproctologia da Faculdade de Ciências Médicas da UNICAMP.

\section{RESUMO}

Objetivo: Avaliar o efeito das aderências dirigidas sobre a resistência mecânica de anastomoses realizadas em cólon isquêmico através da aplicação dos testes Resistência à Pressão de Explosão e Resistência à Força de Tração. Métodos: Quarenta ratos foram distribuídos igualmente em dois grupos. O Grupo 1 era constituído de vinte animais em que as anastomoses cólicas isquêmicas foram recobertas por uma película de polivinilcloreto (P.V.C.) para impedir a formação de aderências sobre as linhas de sutura e o Grupo 2 era constituído por 20 animais em que as anastomoses cólicas isquêmicas foram recobertas pela gordura epididimal, de forma a constituir uma aderência dirigida sobre a linha de sutura, sendo ainda recobertas por uma película de P.V.C. para impedir a formação de outras aderências. Resultados: A média da Pressão de Ruptura à Distensão por Líquidos da anastomose foi de 10 mmHg no grupo sem aderência e de 55,2 mmHg no grupo com aderência $(p<0,05)$. A média da Força de Ruptura à Tração das anastomoses foi de 142,5 gf no grupo sem aderências e de 262 gf no grupo com aderência $(p<0,05)$. Conclusão: As aderências mostraram ser benéficas, elevando a resistência intrínseca das anastomoses tanto na Resistência à Distensão por Líquido quanto na Resistência à Força de Tração.

Descritores: Anastomose Cirúrgica. Cólon. Isquemia. Animais de Laboratório.

\begin{abstract}
Purpose: Evaluate the effect of conducted adhesions on the mechanical resistance of anastomoses performed in ischemic colon through the Bursting Strength Test and Breaking Strength Test. Methods: Forty rats were divided into two groups. Group 1 was composed of 20 rats in which the anastomoses were covered with P.V.C. (Polyvinyl Chloride) to prevent the occurrence of adhesions over the anastomotic line. Group 2 was composed of 20 rats in which anastomotic line was covered with epididimal fat besides P.V.C. to prevent formation of other adhesions. Results: The mean Bursting Strength of the anastomoses in the group without adhesion was $10 \mathrm{mmHg}$ and this value was $55.2 \mathrm{mmHg}$ in the group with adhesion ( $\mathrm{p}<0.05$ ). The mean Breaking Strength of the anastomoses was $142.5 \mathrm{gf}$ in the group without adhesion and $262 \mathrm{gf}$ in the group with adhesion $(\mathrm{p}<0.05)$. Conclusion: Based on those mechanical tests, adhesions were benefic to ischemic anastomoses, rising their intrinsic resistance both on Bursting and Breaking Strength.
\end{abstract}

Key words: Anastomosis, Surgical. Colon. Ischemia. Animals, Laboratory.

\section{Introdução}

A deiscência de uma anastomose intestinal ainda hoje constitui um dos maiores problemas nos intervenções cirúrgicas gastrintestinais e diversos trabalhos foram desenvolvidos com enfoque nos fatores que interferem com o processo de cicatrização das anastomoses cólicas ${ }^{1 \mathrm{a} 27}$. A utilização de aderências dirigidas como "reforço” de uma anastomose faz parte do arsenal técnico do cirurgião e parecem reduzir a ocorrência de necrose, proporcionando uma vedação das falhas nas linhas de anastomoses, além de aumentar a capacidade de drenagem linfática ${ }^{28,29}$, proporcionando uma vedação de falhas nas linhas de anastomoses e aumento na capacidade de drenagem linfática ${ }^{29-33}$. Existem poucos trabalhos experimentais que demonstrem a influência dessas aderências em anastomoses de cólon isquêmico. Neste trabalho realizou-se ensaios mecânicos com o objetivo de avaliar a ação da aderência dirigida sobre anastomoses cólicas isquêmicas. 


\section{Métodos}

Foram utilizados 40 ratos (Rattus norvegicus albinus, Rodentia mammalia) da linhagem Wistar, machos, com peso variando entre 285 a 340 gramas, com idade aproximada de 40 dias, que foram fornecidos pelo Biotério Central / CEMIBUNICAMP. Todos os ratos foram operados pelo autor. Não houve nenhum preparo pré-operatório. Os animais tiveram livre acesso à ração e água durante o período experimental.

Os animais foram anestesiados com pentobarbital sódico em solução com cinco miligramas por mililitro, por punção da veia caudal, e após, esses eram submetidos à laparotomia por incisão mediana. O cólon dos ratos era lavado com solução fisiológica através de punção do ceco por meio de um cateter de Teflon ${ }^{\circledR}$ endovenoso número 16 até a completa limpeza mecânica macroscópica do cólon. $\mathrm{O}$ orifício da punção era fechado com sutura em bolsa utilizando-se fio de polipropileno 7-0. No sigmóide, a dois centímetros da reflexão peritoneal, delimitava-se o local para a realização de secção e anastomose. Esse segmento de alça era submetido à isquemia por ligadura da arcada vascular cólica junto à flexura esplênica, do tronco da artéria mesentérica caudal e da arcada vascular cólica junto à reflexão peritoneal. Após a ligadura dos vasos, eram colocados clampes vasculares, sendo um proximal e outro distal ao local previamente demarcado de secção do cólon sigmóide. Após esses procedimentos, a alça era secionada transversalmente utilizando-se a tesoura de íris. Os segmentos eram anastomosados em plano único, seromuscular, extramucoso com justaposição de bordas e 25 pontos, em média, com fio de polipropileno sete zeros, munido de agulha cardiovascular de um centímetro por técnica biangular.

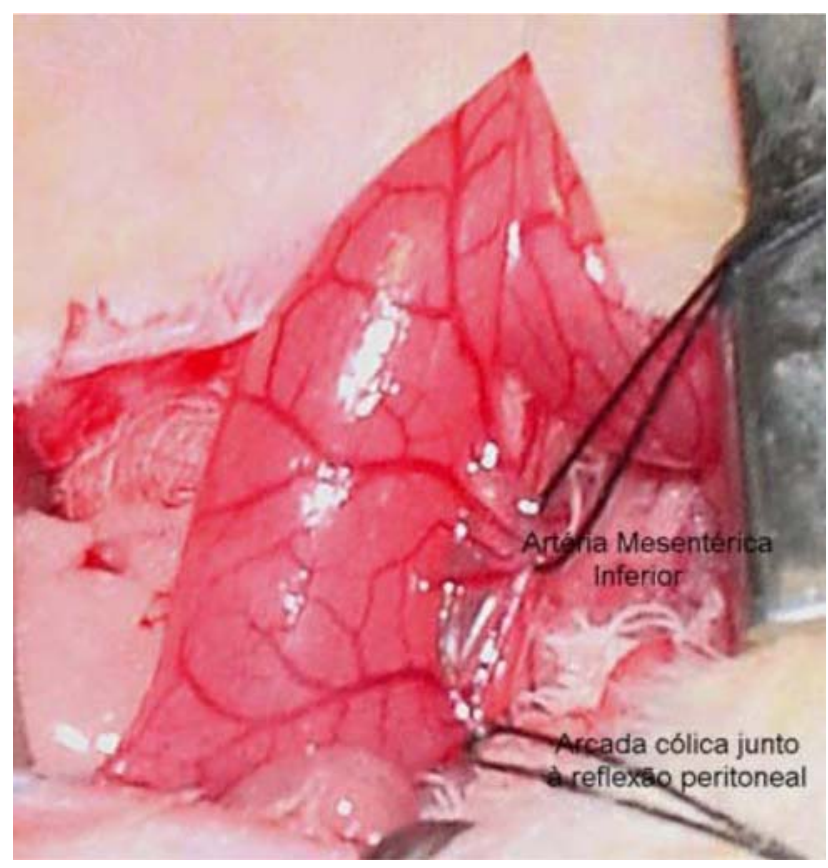

FIGURA 1 - Ligadura do tronco da artéria mesentérica caudal e da arcada cólica junto à reflexão peritoneal.

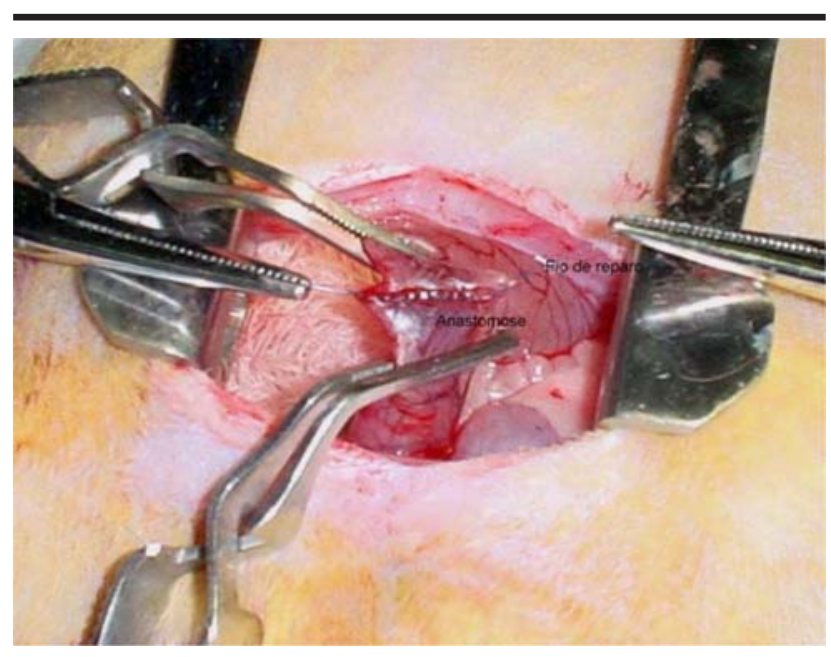

FIGURA 2 - Anastomose completada com clampes vasculares próximos à área de secção do cólon e fios de polipropileno sete zeros utilizados como reparos para técnica biangular.

Terminada a confecção da anastomose, os ratos eram divididos em dois grupos compostos por 20 ratos cada um através de sorteio. As anastomoses dos ratos do Grupo 1 eram recobertas com uma película de polivinilcloreto (P.V.C.) recortada em forma quadrada, com o objetivo de impedir a formação de aderências ao redor da anastomose. As anastomoses dos ratos do Grupo 2 eram envolvidas, em sua circunferência, com a gordura epididimal e depois recobertas com uma película de P.V.C.. Nos dois grupos, estruturas como epíploon e vesículas seminais eram fixadas à película de P.V.C. com o intuito de evitar a formação de aderências em área de anastomose. Após esses procedimentos, a cavidade abdominal era fechada por planos. Recuperados da anestesia, os ratos tiveram livre acesso à alimentação e água durante o período pósoperatório e eram colocados em gaiolas de contenção e mantidos no Biotério do Núcleo de Medicina e Cirurgia Experimental da UNICAMP.

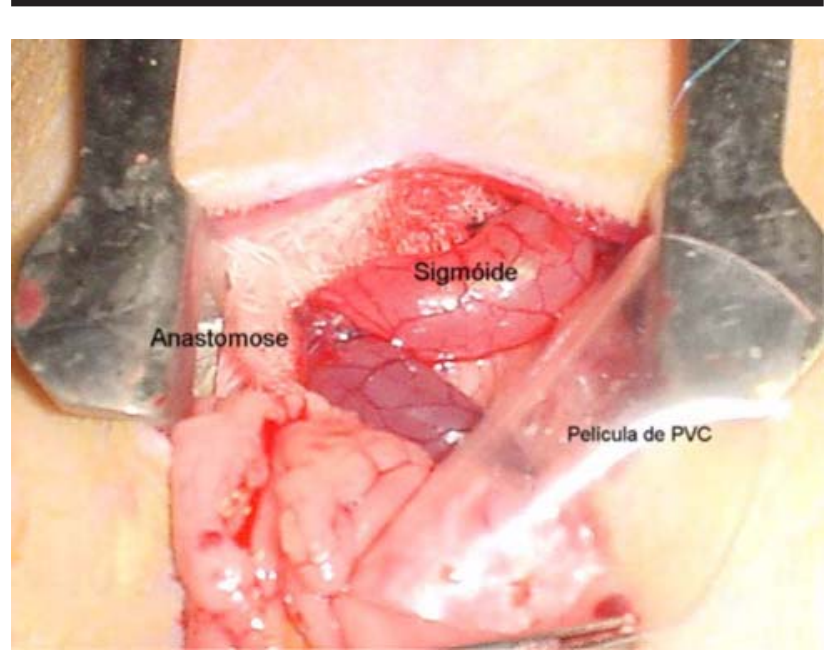

FIGURA 3 - Colocação de película de PVC sobre a anastomose cólica. 
Os ratos foram submetidos à eutanásia no quarto dia pós-operatório por injeção letal de pentobarbital sódico a três por cento administrada pela veia caudal. A parede abdominal era aberta por incisão em U-invertido. As aderências sobre a película de P.V.C. eram removidas e o segmento cólico contendo a anastomose era cuidadosamente retirado, deixando-se margens de dois centímetros proximal e distal à área de sutura. O segmento retirado era então submetido à limpeza cuidadosa do lúmen, retirando-se as fezes, quando presentes, com solução fisiológica aquecida a $37^{\circ} \mathrm{C}$ e colocado em recipiente individual com solução, aquecida à $37^{\circ} \mathrm{C}$, de papaverina em concentração de 250 miligramas por litro por um período de 30 minutos.

Os segmentos cólicos foram submetidos aos testes Pressão de Ruptura à Distensão por líquido e Força de Ruptura à Tração. O teste Pressão de Ruptura à Distensão por líquido foi definido como a pressão intraluminar necessária para ocasionar a ruptura do segmento cólico estudado. O ensaio era realizado pela infusão contínua e constante de solução fisiológica com o auxílio de uma bomba de infusão, através de uma das extremidades da alça (2 mililitros/segundo). A pressão foi registrada por intermédio de um polígrafo, em milímetros de mercúrio. O segundo teste foi definido como a força máxima aplicada em sentido axial necessária para romper a linha anastomótica do segmento de alça. Essa força foi medida em gramasforça através de uma célula de carga de uma balança de precisão acoplada a um computador. Os dados emitidos por esse componente eram captados e convertidos em gráfico por meio do computador, com o auxílio do programa SABI - Sistema de Aquisição e Análise de Dados Biomecânicos. Esse aplicativo foi desenvolvido em parceria pelo LABI - Laboratório de Bioinformática da Unioeste Campus de Foz do Iguaçu e o serviço de Coloproctologia da UNICAMP.

\section{Resultados}

Após a eutanásia, os dois grupos estudados apresentavam, macroscopicamente, características inflamatórias semelhantes. A película de P.V.C. apresentavase igualmente translúcido nos dois grupos. No Grupo 1 havia apenas uma película de fibrina ao redor das anastomoses, sem outras aderências. No Grupo 2, havia uma película de fibrina mais delgada em relação ao Grupo 1 e essa estrutura era visível ao redor da gordura utilizada para envolver a anastomose. Não ocorreram aderências diferentes das programadas em nenhum dos grupos

Vinte ratos, sendo 10 do Grupo 1 (sem aderências dirigidas - Subgrupo 1.1) e 10 do Grupo 2 (com aderências dirigidas - Subgrupo 2.1), foram submetidos ao teste Pressão de Ruptura à Distensão por Líquido. Os valores obtidos estão distribuídos conforme a Tabela 1.

Os dados da Tabela 1 foram transformados em formato gráfico (Figura. 4) para melhor compreensão.

Vinte ratos, sendo 10 do Grupo 1 (sem aderências dirigidas - Subgrupo 1.2) e 10 do Grupo 2 (com aderências dirigidas - Subgrupo 2.2) foram submetidos ao teste Força de Ruptura à Tração. Os dados obtidos estão representados na Tabela 2. Os dados da Tabela 2 estão distribuídos conforme representação gráfica abaixo (fig. 5).

Os testes estatísticos t-Student e Mann-Whitney foram aplicados para comparar os subgrupos com e sem aderência nos testes Pressão de Ruptura à Distensão por Líquido e Força de Ruptura à Tração e mostraram diferença significante entre os grupos com e sem aderências dirigidas nos dois testes $(\mathrm{p}<0,05)$, sugerindo que as aderências dirigidas em cólon isquêmico aumentam a resistência intrínseca da linha de sutura, promovendo efeito protetor sobre as anastomoses cólicas isquêmicas.

TABELA 1 - Resultados do teste Pressão de Ruptura à Distensão por Líquidos.

\begin{tabular}{lllllllllllll}
\hline Grupos/Ratos & $\mathbf{1}$ & $\mathbf{2}$ & $\mathbf{3}$ & $\mathbf{4}$ & $\mathbf{5}$ & $\mathbf{6}$ & $\mathbf{7}$ & $\mathbf{8}$ & $\mathbf{9}$ & $\mathbf{1 0}$ & Média & Desvio padrão \\
\hline $\begin{array}{l}\text { Sem aderências } \\
\text { (SubGrupo 1.1) }\end{array}$ & 2 & 2 & 3 & 5 & 6 & 10 & 12 & 14 & 18 & 28 & 10 & 8,34 \\
\hline $\begin{array}{l}\text { Com aderências } \\
(\text { SubGrupo 2.1) }\end{array}$ & 20 & 40 & 40 & 44 & 56 & 60 & 60 & 68 & 72 & 92 & 55,2 & 20,2 \\
\hline
\end{tabular}

TABELA 2 - Resultados do teste Força de Ruptura à Tração.

\begin{tabular}{lllllllllllll}
\hline Grupo/Ratos & $\mathbf{1}$ & $\mathbf{2}$ & $\mathbf{3}$ & $\mathbf{4}$ & $\mathbf{5}$ & $\mathbf{6}$ & $\mathbf{7}$ & $\mathbf{8}$ & $\mathbf{9}$ & $\mathbf{1 0}$ & Média & Desvio padrão \\
\hline $\begin{array}{l}\text { Sem aderências } \\
\text { (Subgrupo 1.2) }\end{array}$ & 74 & 74 & 100 & 141 & 165 & 165 & 170 & 175 & 176 & 185 & 142,5 & 43,5 \\
\hline $\begin{array}{l}\text { Com aderências } \\
(\text { Subgrupo 2.2) }\end{array}$ & 225 & 228 & 249 & 252 & 252 & 263 & 270 & 270 & 304 & 307 & 262 & 27,4 \\
\hline
\end{tabular}


Pressão de Explosão sem e com aderências dirigidas

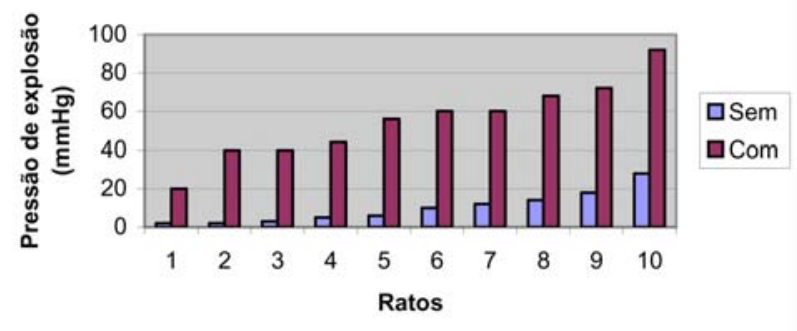

FIGURA 4 - Representação gráfica dos dados da Tabela 1.

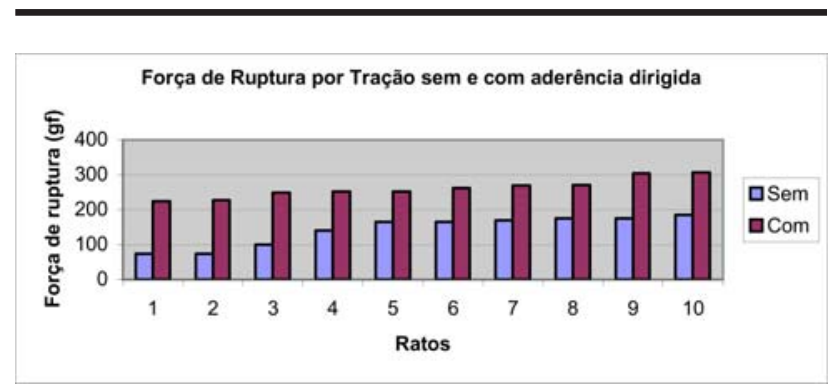

FIGURA 5 - Rsentação gráfica dos dados da Tabela 2.

\section{Discussão}

Diversos fatores, como deficiência nutricional ${ }^{4}$, distúrbios metabólicos ${ }^{5}$, processos inflamatórios e infecciosos ${ }^{18}$, agentes farmacológicos ${ }^{7,26}$ e formação de aderências $^{27}$, interferem no processo de cicatrização intestinal. Trabalhos experimentais em áreas biológicas são influenciados por uma série de fatores que repercutem na precisão dos resultados. Com o objetivo de reduzir as influências exercidas pela variabilidade biológica, utilizouse animais que permitem estabelecer uma similaridade fisiológica, anatômica, cronológica e ponderal ao grupo que será estudado.

Em uma série as aderências foram responsáveis por $32 \%$ dos casos de obstrução intestinal ${ }^{27}$, o que levou vários autores a estudarem maneiras de impedir sua formação ${ }^{28,29,30}$. As ações das aderências, entretanto, não são sempre prejudiciais, pois essas estruturas podem funcionar como mecanismos protetores por meio de tamponamento mecânico em eventuais áreas de falha de anastomose. Além disso, essas estruturas proporcionam neovascularização na linha de sutura, aumentando a capacidade fagocitária e drenagem linfática ${ }^{22-25}$.

A utilização de aderências dirigidas há muito tempo faz parte do arsenal técnico dos cirurgiões. Senn ${ }^{30}$, após experimentos em cães, demonstrou que o grande omento deveria ser utilizado para proteger anastomoses intestinais. Wu $^{31}$ estudou o comportamento mecânico das aderências dirigidas em ratos, concluindo que as aderências abdominais formadas fisiologicamente exercem influência no aumento da resistência sobre as anastomoses cólicas. Neste trabalho dois grupos foram formados, sendo o Grupo 1 representado pela anastomose sem aderências dirigidas e o Grupo 2, anastomose com aderências dirigidas. Esses grupos foram assim formados com o objetivo de permitir a avaliação da influência de aderências sobre a linha de anastomose em cólon isquêmico. A Pressão de Ruptura à Distensão é um teste de resistência à insuflação por gás ou líquido, o qual permite avaliar a pressão necessária para romper, de modo puntiforme ou parcial, uma anastomose $e^{9,30-32,38}$. Variáveis como o tempo decorrido entre o sacrifício e o teste, a extensão dos segmentos intestinais, o diâmetro do segmento intestinal, o espasmo dos segmentos intestinais (fisiológico ou decorrente do manuseio), o dia pós-operatório em que os segmentos foram estudados e a velocidade de infusão do líquido podem interferir com o resultado desse teste. Entretanto, quando esses resultados são obtidos dentro de um mesmo estudo, com animais possuindo características homogêneas, seguindo um protocolo experimental, as comparações dos resultados são válidos ${ }^{32}$. O método Pressão de Ruptura à Distensão só tem validade quando a ruptura ocorre na linha de sutura. Esse critério foi adotado neste trabalho.

A Força de Ruptura à Tração permite analisar a resistência de uma anastomose quando esta é submetida à força variável com o tempo aplicada em sentido axial, perpendicular em relação à linha de sutura. Foi utilizada por vários pesquisadores. ${ }^{18,34,39-41}$ Essa análise pode ser realizada testando-se toda a anastomose ou fragmentos dela. A utilização de fragmentos contendo parte da linha anastomótica pode trazer dificuldades de padronização. Além disso, essas tiras de tecido contendo parcialmente a linha de sutura, não representam a anastomose na sua integralidade, dificultando a correlação dos resultados obtidos. Assim como ocorre na Pressão de Ruptura à Distensão, a Força de Ruptura à Tração pode sofrer influência de vários fatores.

\section{Conclusão}

As aderências dirigidas aumentaram de forma significativa a resistência mecânica de anastomoses em cólon isquêmico de ratos. Estudos sobre o mecanismo de ação das aderências sobre o processo de cicatrização ainda precisam ser realizados.

\section{Referências}

1. Goligher JC, Graham NG, De Dombal FT, Johnston D. A controlled trial of inverting versus everting intestinal suture in clinical large-bowel surgery. Br J Surg. 1970b;57:817-22

2. Abramowitz HB, Butcher Júnior HR. Everting and inverting anastomoses: an experimental study of comparative safety. Am J Surg. 1971;121:52-6.

3. Leonardi LS. Resultado do emprego da sutura em plano único extramucoso na cirurgia gástrica [Tese De Livre Docência]. Universidade Estadual de Campinas Faculdade de Ciências Médicas; 1973.

4. Aprilli F, Guimarães AS, Rocha JJR. Anastomoses Intestinais. In: Silva Júnior, OC - Modelos experimentais de pesquisa em cirurgia. Capítulo 38, 1ed. Robe Editorial. São Paulo, 1998.

5. Goodson WHHH, Hunt TK. Wound healing in experimental diabetes mellitus: importance of early insulin therapy. Surg Fórum. 1978;29:95-8. 
6. Mantovani M, Leonardi LS, Alcântara FG, Medeiros RR, Fagundes JJ, Hadler WA. Estudo comparativo entre diferentes variedades de suturas em um e dois planos no intestino delgado. Trabalho experimental No Cão. Rev Assoc Med Bras. 1976;22:79-86.

7. Mantovani M. Evolução da cicatrização em anastomoses do intestino grosso de cães em condições de normalidade e sob a ação de drogas imunossupressoras [Tese De Livre Docência]. Universidade Estadual de Campinas Faculdade de Ciências Médicas; 1978.

8. Jiborn H, Ahonen J, Zederfeldt B. Healing of experimental colonic anastomoses. i. bursting strength of the colon after left colon resection and anastomosis. Am J Surg. 1978a;136:587-94.

9. Jiborn H, Ahonem J, Zederfeldt B. Healing of experimental colonic anastomoses. ii. breaking strength of the colon after left colon resection and anastomosis. Am J Surg. 1978b;136:595-9.

10. Jiborn H, Ahonen J Zederfeldt B. Healing of experimental colonic anastomoses. collagen metabolism in the colon after left colon resection. Am J Surg. 1980a;139:398-405.

11. Jiborn H, Ahonen J, Zederfeldt B. Healing of experimental colonic anastomoses. Effect of suture technique on collagen metabolism in the colonic wall. Am J Surg. 1980b;139:406-13.

12. Lord MG, Broughton AC, Willians HTG. A morphologic study on the effect of suturing the submucosa of the large intestine. Surg Gynecol Obstet. 1978;146:211-6.

13. Kobayasi S, Mendes EF. Estudo clínico prospectivo entre suturas contínuas em plano único e em dois planos, em gastrojejunoanastomoses. Rev Paul Med. 1982;100:11-4.

14. Ballantyne GH. Intestinal suturing. Review of experimental foundations for traditional doctrines. Dis Colon Rectum. 1983;26:836-43.

15. Khoury GA, Waxmann BP. Large bowell anastomoses. I. The healing process and suture anastomoses. A Review. Br J Surg. 1983;70:61-3.

16. Houdart R, Lavergne A, Valleur P, Villet R, Hautefeville P. Vascular evolution of single-layer end on colonic anastomosis. A microangiographic study of 180 anastomoses in the rat from two to 180 days. Dis Colon Rectum. 1985;28:475-80.

17. Fagundes JJ. Estudo comparativo da cicatrização de anastomoses cólicas realizadas com auxílio do bisturi laser de dióxido de carbono: trabalho experimental em cães [Tese De Doutorado]. Universidade Estadual de Campinas - Faculdade de Ciências Médicas; 1990.

18. Naresse LE. Efeito da peritonite fecal na cicatrização do cólon distal no rato. Avaliação anátomo-patológica, estudo da força de ruptura e da hidroxiprolina tecidual [Tese De Doutorado]. Universidade Estadual Paulista Botucatu - Faculdade de Ciências Médicas; 1990.

19. Santos Júnior JCM. Profilaxia das complicações pósoperatórias no tratamento cirúrgico das doenças do intestino grosso: i- deiscência da anastomose. Rev Bras Coloproctol. 1998;18:44-51.

20. Nieri TM. Estudo sobre o comportamento mecânico do cólon íntegro e com anastomose. Trabalho experimental em rato [Tese de Mestrado]. Universidade Estadual de Campinas - Faculdade de Ciências Médicas; 1990.
21. Myllarniemi H, Karppinen V. Vascular pattern of peritoneal adhesions. Br J Surg. 1968;55:605.

22. Ellis H. Wound repair- reaction of the peritoneum to injury. Ann R Coll Surg. 1978;60:219-21.

23. Ellis H. The causes and prevention of intestinal adhesions. Br J Surg. 1982;69:241-3.

24. Christen D, Buckmann RF. Peritoneal Adhesions After Laparotomy: Prophylatic Mesures. Hepatogastroenterology. 1991;38:283-6.

25. Lanter B, Mason RA. Use of omental pedicle graft to protect low anterior colonic anastomosis. Dis Colon Rectum. 1979;22:448-51.

26. Minossi JG. Ação do diclofenaco de sódio na cicatrização de anastomoses realizadas no íleo terminal e no cólon distal de ratos. Estudo da força de ruptura, hidroxiprolina tecidual e exame histopatológico [Tese De Doutorado]. Universidade Estadual Paulista - Botucatu - Faculdade de Ciências Médicas;1995.

27. Mcentee G, Pender D, Mulvin D, Mccullough M. Current spectrum of intestinal obstruction. Br J Surg. 1987;74:976-80.

28. Aulusia B, Gazzaniga M, Beretta T. The effect of fibrinolytic substance in prevention and treatment of post-operative peritoneal adhesions. Panminerva Med. 1966;8:432.

29. Polishuk WZ, Aboulafia Y. Dextran in prevention of peritoneal adhesions. Isr J Med Sci. 1967;3:806.

30. Senn N. An experimental contribution to intestinal surgery with special reference to the treatment of intestinal obstruction. Ann Surg. 1888;7:421.

31. Wu FC estudo da ação de aderências sobre anastomose cólica: trabalho experimental em ratos [Tese De Mestrado]. Universidade Estadual de Campinas Faculdade de Ciências Médicas; 2000.

32. Cronin K, Jackson DS, Dunphy JE. Changing bursting strength and collagen content of the healing colon. Surg Gynecol Obstet. 1968;126:747-53.

33. Carril CF. Anastomoses intestinais: métodos invaginantes e métodos por aposição em mesmo alinhamento. Estudo experimental comparativo com apresentação de um processo original [Tese De LivreDocência]. Universidade de São Paulo - Ribeirão Preto - Faculdade de Medicina; 1970.

34. Brennam SS, Póster ME, Morgan A, Leaper DJ. Prostaglandins in colonic anastomotic healing. Dis Colon Rectum. 1984;27:723-5.

35. Foster ME, Johnson CD, Billings PJ, Davies PW, Leaper DJ. Intraoperatíve antegrade lavage and anastomotic healing in acute colonic obstruction. Dis Colon Rectum. 1986;29:255-9.

36. Rocha JJR. Estudo comparativo entre sutura contínua e sutura com pontos separados em anastomoses colocólicas em plano único. Trabalho experimental em cães [Tese De Doutorado]. Universidade de São Paulo Ribeirão Preto - Faculdade de Medicina; 1989.

37. Biondo-Simões MLP, Koppe GL, Hansell H, Rasário MAK, Malafaia O. Uso de adesivo biológico em anastomoses intestinais. Estudo experimental em cães. Acta Cir Bras. 1992;7:151-3.

38. Herrmann JB, Woodward SC, Pulaski EJ. Healing of colonic anastomoses in the rat. Surg Gynecol Obstet. 
1964;119:169-75.

39. Blomquist P, Jiborn H, Zederjfelt B. The effect of relative bowel rest on healing of colonic anastomoses: breaking strength and collagen in the colonic wall following left colon resection and anastomoses in the rat. Acta Chir Scand. 1984;150:671-5.

40. Uden P, Blomquist P, Jiborn H, Zederfelt B. Influence of proximal colostomy on the healing of a left cólon anastomosis: an experimental study in the rat. Br J Surg. 1988;75:325-9.
41. Leite CVS, Naresse LE, Saad LHC, Thomazini I, Barravieira B, Kobayasi S. Cicatrização intestinal - efeito da cola derivada de veneno de cobra, na anastomose de cólon em ratos. Rev Bras Coloproctol. 1994;14 (Supl.):24.

\section{Agradecimentos}

Agradecemos aos Biólogos do Núcleo de Medicina e Cirurgia Experimental da Unicamp, Ana Cristina de Moraes e William Adalberto Silva. Agradecemos também ao Laboratório de Bioinformática da UNIOESTE.

\section{Correspondência:}

Miki Mochizuki

Rua Rafael Aloisi, 62

13405-205 Piracicaba - SP

gastromiki@yahoo.com
Conflito de interesse: nenhum Fonte de financiamento: nenhuma

Recebimento: 18/01/2005

Revisão: 23/02/2005

Aprovação: 29/03/2005

\section{Como citar este artigo:}

Mochizuki M, Wu FC, Coy CSR, Ayrizono MLS, Góes JRN, Fagundes JJ. Efeito de aderências dirigidas em anastomoses cólicas isquêmicas em ratos. Acta Cir Bras. [periódico na Internet] 2005 Maio-Jun;20(3). Disponível em URL: http://www.scielo.br/acb

*Figuras coloridas disponíveis em http://www.scielo.br/acb 